Document downloaded from:

http://hdl.handle.net/10251/83351

This paper must be cited as:

Sánchez-Escuderos, D.; Ferrando Bataller, M.; Herranz Herruzo, JI.; Rodrigo Peñarrocha, VM. (2016). Low-loss Circularly Polarized Periodic Leaky-Wave Antenna. IEEE Antennas and Wireless Propagation Letters. 15:614-617. doi:10.1109/LAWP.2015.2463672.

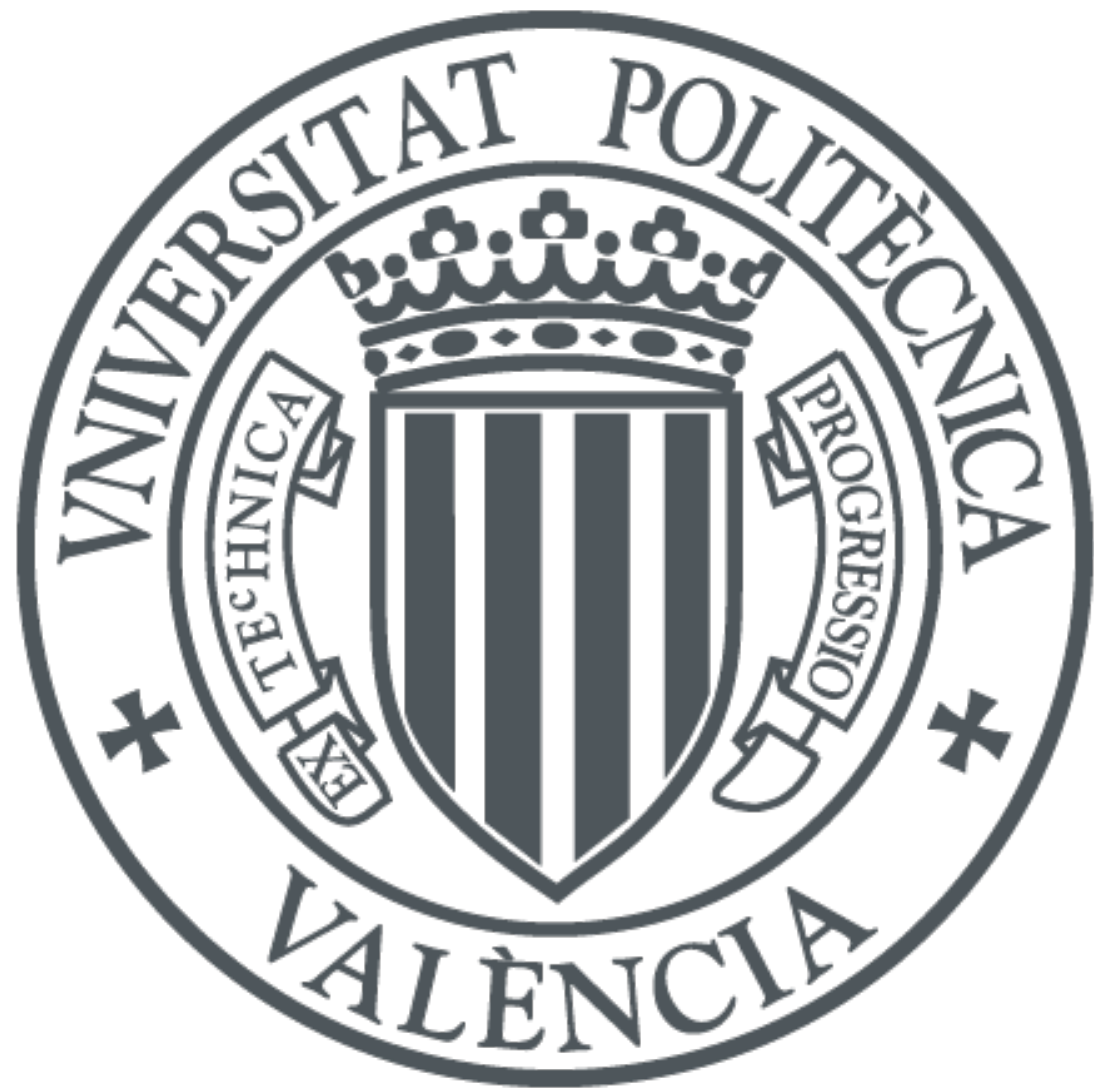

The final publication is available at

http://dx.doi.org/10.1109/LAWP.2015.2463672

Copyright Institute of Electrical and Electronics Engineers (IEEE)

Additional Information

(c) 2015 IEEE. Personal use of this material is permitted. Permission from IEEE must be obtained for all other users, including reprinting/ republishing this material for advertising or promotional purposes, creating new collective works for resale or redistribution to servers or lists, or reuse of any copyrighted components of this work in other works 


\title{
Low-loss Circularly Polarized Periodic Leaky-Wave Antenna
}

\author{
Daniel Sánchez-Escuderos, Member, IEEE, Miguel Ferrando-Bataller, Member, IEEE, \\ Jose I. Herranz, Member, IEEE, Vicent Miquel Rodrigo-Peñarrocha.
}

\begin{abstract}
This letter presents a periodic leaky-wave array antenna with circular polarization at millimeter-wave frequencies. The antenna is designed on the low-loss planar Goubau transmission line to maximize the radiation efficiency of the antenna. The unit cell of the array is formed by four dipoles located on both faces of the Goubau line' substrate. A prototype has been fabricated and measured. Experimental results show an impedance bandwidth above $15 \%$ for $\left|S_{11}\right|<-10 \mathrm{~dB}$ and a 3-dB axial ratio bandwidth of $7.6 \%$. The radiation efficiency and maximum gain of the prototype are above $90 \%$ and $15.6 \mathrm{dBi}$, respectively, from 38 to $41 \mathrm{GHz}$. The maximum gain and the minimum axial ratio present the characteristic steering behavior of leaky-wave antennas.
\end{abstract}

Index Terms-Circularly polarization, leaky-wave antennas, millimeter-wave antenna arrays, antenna efficiency.

\section{INTRODUCTION}

$\mathbf{T}$ HE demand for circularly polarized antennas has experienced a considerable increment during last years. On the one hand, circularly polarized (CP) waves are less sensitive to the Faraday rotation in the ionosphere [1], what has caused the extensive use of $\mathrm{CP}$ antennas in satellite communication systems [2] and GPS [3]. On the other hand, CP antennas minimize the polarization mismatch and the multi-path interference problems in radar [4] and wireless communication systems [5]. These advantages become specially useful at millimeter-wave frequencies to mitigate their high free-space losses [6].

In order to increase the gain of a $\mathrm{CP}$ antenna, an array of elements with circular polarization is commonly used. The array bandwidth is strongly dominated by the power distribution network. Whereas a series feeding presents a reduced bandwidth [7], a corporate feeding widens the bandwidth by equalizing the feeding path to each element [8], at the expense of increasing the size of the structure and the ohmic losses.

Periodic leaky-wave antennas are a special case of seriesfed array antennas. The elements of these antennas are fed sequentially, and spaced by either more or less than onehalf wavelength. This separation, together with the absence of a final short circuit, confers these antennas a wideband performance and a frequency-steerable radiation pattern.

Leaky-wave antennas are often designed in microstrip technology due to its low profile, light weight and low production

This work has been supported by the Spanish Ministry of Education and Science (Ministerio de Educacion y Ciencia) under the projects TEC201020841-C04-01, TEC2013-47360-C3-3-P and CSD2008-00068

The authors are with the Instituto de Telecomunicaciones y Aplicaciones Multimedia (ITEAM) of the Universitat Politècnica de València (UPV), Cami de Vera s/n, 46022, Valencia, Spain (e-mail: dasanes1@iteam.upv.es; mferrand@dcom.upv.es; jiherhe@upvnet.upv.es, vrodrigo@dcom.upv.es).

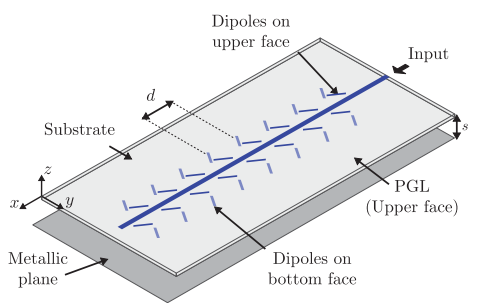

(a)

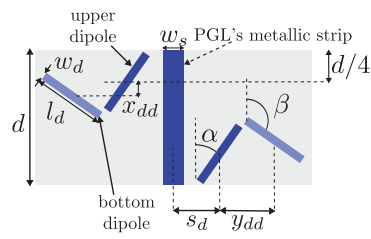

(b)
Fig. 1. Circularly polarized periodic leaky-wave antenna on planar Goubau line: (a) complete model and (b) unit cell.

cost [9]. The drawback of this technology resides in the inherent low radiation efficiency of the resulting antenna [9], [10], mainly caused by the high losses in the microstrip line. Recently, a leaky-wave array fed by a Planar Goubau Line (PGL) has been proposed with the aim to reduce losses and, consequently, maximize the radiation efficiency [11].

This letter proposes a new periodic leaky-wave array antenna with circular polarization on a PGL. The perturbations are formed by two orthogonal dipoles located on different faces of the PGL's dielectric substrate to improve the polarization purity.

The proposed antenna is formed by 16 perturbations, distributed alternatively on both sides of the PGL's central metallic strip. A reflector metallic plane is additionally introduced to suppress the downward beam and achieve an upward single main beam. A prototype has been manufactured and measured.

\section{CIRCUlARLY POLARIZED PERIODIC LEAKY-WAVE ANTENNA}

A sketch of the proposed circularly polarized periodic leaky-wave antenna (CP-PLWA) is shown in Fig. 1 (a). As commented above, the antenna is designed using a PGL as a power distribution line. The PGL, formed by a metallic strip on a dielectric substrate, is the planar version of the classical Goubau line [12]. Unlike microstrip lines, a ground plane is not present in the PGL and, hence, the confinement of field within the dielectric substrate is minimized. Since the fundamental mode of the PGL is bounded, perturbations must be inserted to produce the desired radiation. This principle was already used in [11] to design a periodic leaky-wave antenna with linear polarization. For the present work, line perturbations must be changed in order to radiate $\mathrm{CP}$ waves. This section describes the unit cell of these perturbations as well as the complete CP-PLWA. 


\section{A. Unit cell: Double dipole}

The unit cell of the CP-PLWA, depicted in Fig. 1 (b), is formed by four dipoles grouped in two sets of orthogonal dipoles with rotation angles $\alpha$ and $\beta\left(\beta=\alpha+90^{\circ}\right)$, similarly to [7]. On each set, one of the dipoles is located on the upper face of the PGL' substrate, together with the PGL's metallic strip, while the other dipole is placed on the bottom face of the substrate. By placing dipoles on different faces of the substrate, a $90^{\circ}$-phase shift between the orthogonal dipoles can be easily achieved, what provides the desired CP radiation. Note that these advantages are only possible because of the non-grounded nature of the PGL.

Each set of dipoles is separated half of the distance $(d)$ between unit cells, and is located on different sides of the PGL's metallic strip to compensate for the $180^{\circ}$-phase difference. The distance $s_{d}$ from the upper dipole to the metallic strip, and the rotation $\alpha$ of the upper dipole determine the amount of perturbed energy in the PGL. The longitudinal and transverse separation between dipoles, $x_{d d}$ and $y_{d d}$, allows to adjust the coupled energy between orthogonal dipoles.

\section{B. Complete CP antenna}

The complete CP-PLWA, see Fig. 1 (a), is formed by eight unit cells separated a distance $d$. The dipoles of the unit cells perturb periodically the field of the PGL and excite space harmonics. These harmonics radiate as long as their propagation coefficient $\left(\beta_{n}\right)$ satisfies the condition $\beta_{n} / k_{0} \leq 1$, where $k_{0}$ is the free-space wavenumber. The propagation coefficient of each harmonic is determined by the separation $d$, and sets the main beam's direction [11]. If a single mode operation, with $n=-1$, is chosen in the leaky-wave antenna, and a separation $d=5.25 \mathrm{~mm}\left(0.7 \lambda_{0}\right)$ is selected, the main beam points towards the generator with an angle of, approximately, $-20^{\circ}$ with respect to the normal axis at $f_{0}=39.5 \mathrm{GHz}$.

The absence of a ground plane would make the CP-PLWA radiate in two opposite beams: an upward beam $(+z)$ with lefthand circular polarization (LHCP) and a downward beam $(-z)$ with right-hand circular polarization (RHCP). This configuration is not practical in most situations, where a single beam is required. For a coherent addition of both beams, a reflector plane is inserted at a distance $s=5 \mathrm{~mm}\left(0.66 \lambda_{0}\right)$ from the dielectric substrate. Under this configuration, the downward beam is reflected towards the same direction as the upward beam and with the same polarization (RHCP). The change of polarization in the downward beam is caused by the $180^{\circ}$ phase shift of the parallel component to the reflector plane of the CP wave [13]. Consequently, a single upward beam with a higher directivity and a single circular polarization, LHCP, is produced. Since there exists an air gap between the substrate and the reflector metallic plane, the field is not confined within the dielectric substrate, unlike antennas in SIW or microstrip technology [7], and the line preserves its low-loss performance.

The antenna has been optimized using Ansys HFSS [14] with the goal of maximizing the gain and minimizing the axial ratio in the main beam at $f_{0}=39.5 \mathrm{GHz}$. In addition, in order to minimize the losses of the antenna, the design aims to limit

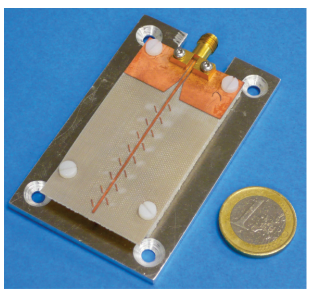

(a)

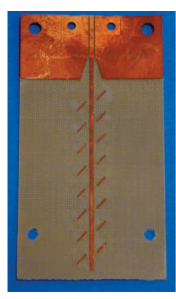

(b)

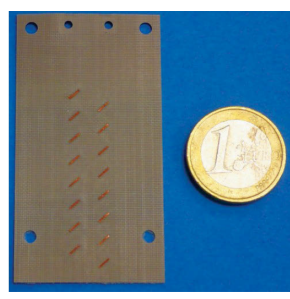

(c)
Fig. 2. Picture of the fabricated prototype: (a) 3D view of the complete CP-PLWA, (b) top view of the upper face of the substrate and (c) top view of the bottom face of the substrate.

the power reaching the end of the antenna. Thus, the use of a final load is avoided.

The optimization was carried out considering a substrate with $\varepsilon_{r}=1.8, \tan \delta=0.02$ and height $h_{s}=508 \mu \mathrm{m}$. The width and thickness of the PGL's metallic strip were $w_{s}=0.75 \mathrm{~mm}$ and $t=35 \mu \mathrm{m}$, respectively. The optimized dimensions of the CP-PLWA are: $\alpha=35^{\circ}\left(\beta=125^{\circ}\right), l_{d}=2.5 \mathrm{~mm}, w_{d}=0.3 \mathrm{~mm}$, $x_{d d}=0.5 \mathrm{~mm}, y_{d d}=2 \mathrm{~mm}$ and $s_{d}=1.7 \mathrm{~mm}$. For the sake of simplicity, all cells have the same dimensions so that the resulting weight distribution along the elements of the CPPLWA is an asymmetrical tapered distribution with a higher weight on the side of the input port.

\section{PRototype AND MEASUREMENTS}

The optimized CP-PLWA has been fabricated with a PCB etching procedure. Fig. 2 shows three pictures of the prototype. The $3 \mathrm{D}$ view of Fig. 2 (a) shows the $2.4 \mathrm{~mm}$ connector used to feed the antenna as well as the coplanar transmission line used to smoothly match the input port impedance $(50 \Omega)$ and the PGL impedance. Fig. 2 (b) and Fig. 2 (c) show the dipoles on the upper and bottom faces, respectively.

In order to assure a constant separation between the dielectric substrate of the antenna and the reflector metallic plate, a $5 \mathrm{~mm}$-thick foam frame is inserted. Thus, an air gap is created between the substrate and the metallic plate, and the high efficiency of the antenna is preserved.

Fig. 3 compares the simulated and measured $S_{11}$ parameter of the CP-PLWA. Results indicate a good matching level in the whole measured band, giving a $-10 \mathrm{~dB}$ impedance bandwidth above $15 \%$. The shift between measured and simulated results may be caused by a small error in the separation $s$ between the substrate and the reflector plane of the prototype, and a bad electrical characterization of the substrate in the Ka-band.

The radiation pattern of the CP-PLWA has been measured at several frequencies within the bandwidth $38-41 \mathrm{GHz}$. Fig. 4 shows the copolar (LHCP) and crosspolar (RHCP) components of the radiation pattern on the $\mathrm{XZ}$ plane (the highest-directivity plane) at $39.5 \mathrm{GHz}$. As it can be seen, the crosspolar component is $15 \mathrm{~dB}$ below the copolar component in the main beam.

Fig. 5 shows the steering behavior of the main beam of the leaky-wave antenna in both, the radiation pattern and the axial ratio (AR). This figure illustrates that, on the one hand, the beamwidth and SLL of the radiation pattern are preserved within acceptable values at all frequencies and, on the other hand, the axial ratio presents a good level within the main 


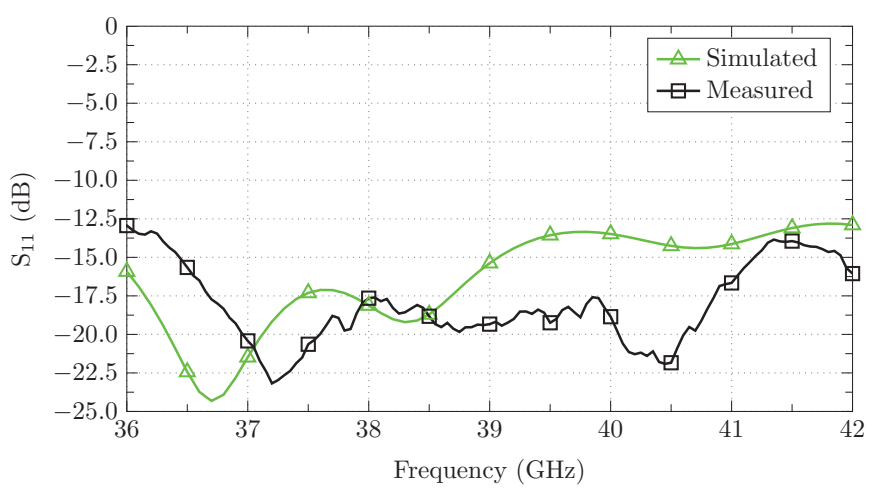

Fig. 3. $\mathrm{S}_{11}$ parameter of the CP-PLWA.

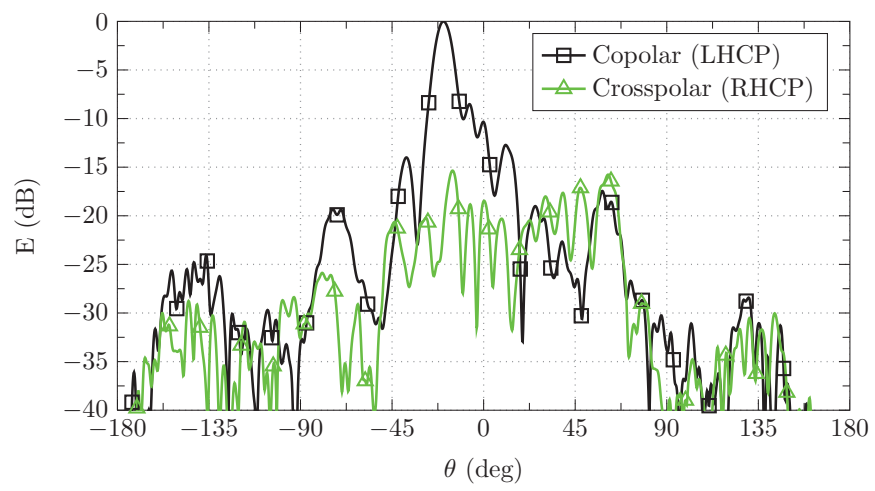

Fig. 4. Copolar and crosspolar components of the radiation pattern in the $\mathrm{XZ}$ plane at $39.5 \mathrm{GHz}$.

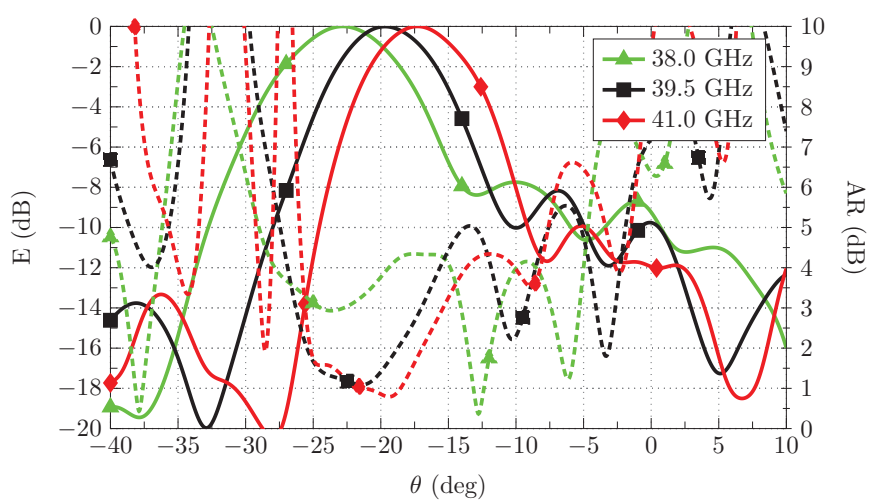

Fig. 5. Measured radiation pattern (solid line) and axial ratio (dashed line) on the $\mathrm{XZ}$ plane at several frequencies.

beam of the antenna at any frequency (thus giving a circular polarization (LHCP) at high gain directions). The main beam at $39.5 \mathrm{GHz}$ points towards $-19.6^{\circ}$. The difference between this direction and the one predicted theoretically is caused by the small modification of $\beta_{0}$ introduced by the perturbations along the line [11].

The measured radiation and AR patterns on the high and low-directive planes at $39.5 \mathrm{GHz}$ are compared to the simulated patterns in Fig. 6 and Fig. 7, respectively. Note that the low directive plane is a plane containing the $y$ axis and the direction of maximum radiation (angle in Fig. 7 represents the elevation angle in this plane). Apart from small

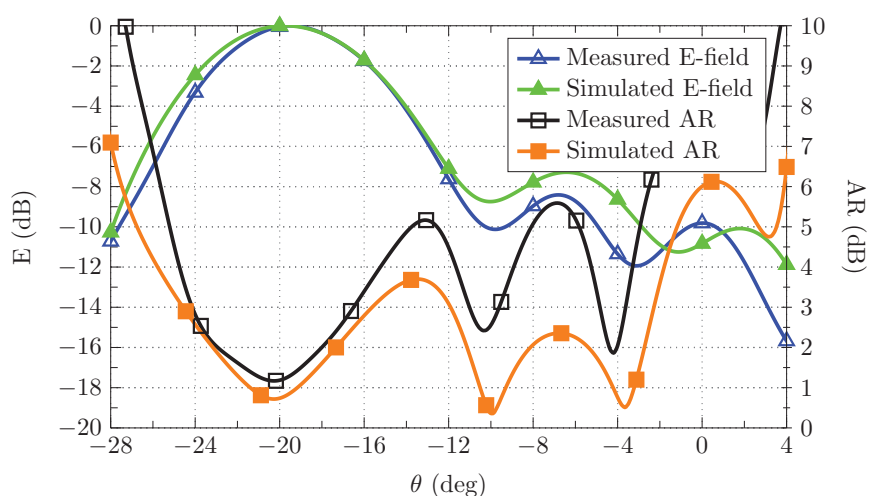

Fig. 6. Comparison of the measured and simulated radiation pattern and axial ratio on the $\mathrm{XZ}$ plane at $39.5 \mathrm{GHz}$.

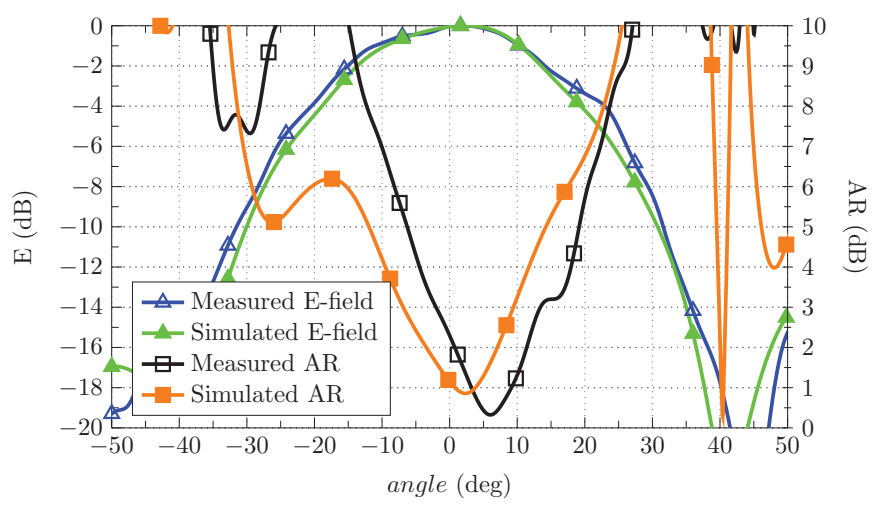

Fig. 7. Comparison of the measured and simulated radiation pattern and axial ratio on the low-directive plane at $39.5 \mathrm{GHz}$.

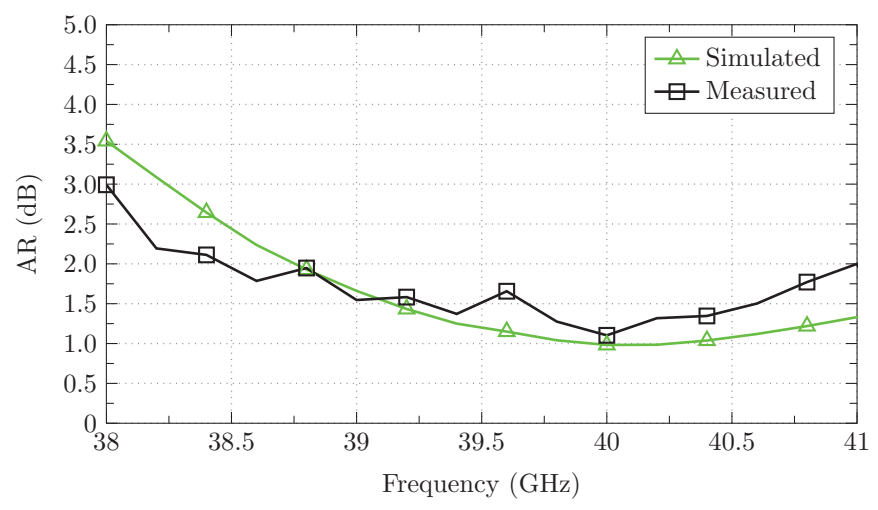

Fig. 8. Axial ratio at maximum radiation angle vs frequency.

differences caused by the same errors that caused the shift in the $S_{11}$ parameter, a good agreement can be observed in this comparison between simulations and measurements. In Fig. 6, it can be verified that the minimum values of the AR coincide with the main beam of the radiation pattern within a half-power beamwidth of $9^{\circ}$.

Fig. 8 represents the measured and simulated AR at the maximum radiation angles across the whole bandwidth 38$41 \mathrm{GHz}$. As can be observed, the AR is below $3 \mathrm{~dB}$ at all frequencies, giving an axial ratio bandwidth above $7.6 \%$.

The gain of the CP-PLWA has been measured in order to 
TABLE I

COMPARISON OF MAIN RESULTS WITH PREVIOUSLY PUBLISHED WORKS.

\begin{tabular}{|c|c|c|c|c|c|}
\hline CP Antennas & Size $\left(\lambda_{0}\right)$ & $-10 \mathrm{~dB}$ RLBW & 3 dB ARBW & Gain & Efficiency \\
\hline$\mu$-strip CP Leaky-wave antenna [10] & $0.94 \times 0.48 \times 10^{-4}$ & $14.28 \%$ & $30.50 \%$ & $1.0 \mathrm{dBi}$ & $30 \%$ \\
\hline X-band planar CP array [15] & $10.66 \times 10.66 \times 0.66$ & $14.70 \%$ & $25.00 \%$ & $26.2 \mathrm{dBi}$ & $60 \%$ \\
\hline CP-PLWA on PGL (This work) & $4.97 \times 0.94 \times 0.66$ & $>15.00 \%$ & $>7.60 \%$ & $15.6 \mathrm{dBi}$ & $93 \%$ \\
\hline
\end{tabular}

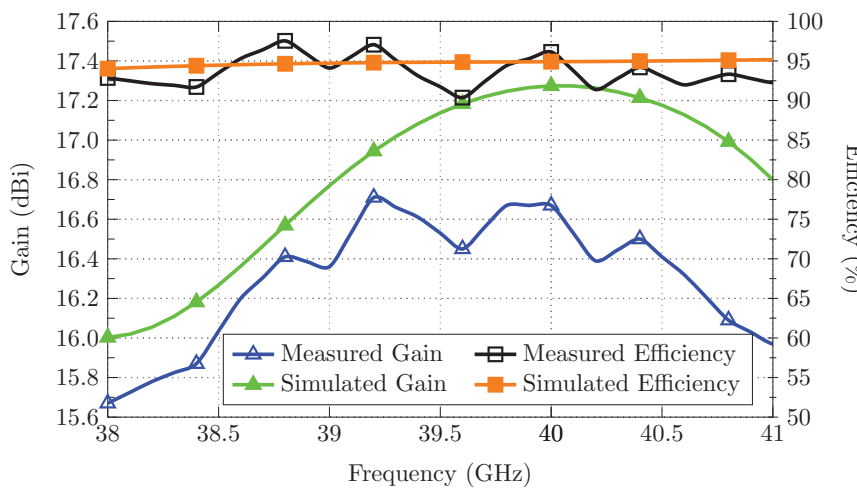

Fig. 9. Maximum gain and efficiency vs frequency of the CP-PLWA.

evaluate the losses of the antenna. To do so, a spherical nearfield measurement set-up with a standard gain horn as a probe and the substitution procedure described in [16] have been implemented. Fig. 9 shows the maximum gain and efficiency of the antenna at different frequencies.

As it can be observed in Fig. 9, the gain is always above $15.6 \mathrm{dBi}$ at all frequencies, with a fluctuation smaller than $1 \mathrm{~dB}$. The efficiency of the antenna is quite high, above $90 \%$ across the whole bandwidth, with an average value of $93 \%$. These high values are caused by the low concentration of field in the dielectric substrate. Note that, in case of designing the CP-PLWA in microstrip technology, the radiation efficiency would have been around 30\%, as shown in [10], [11]. Also, a higher efficiency than in the linearly-polarized array [11] is observed due to a higher weight of the asymmetrical tapered distribution near the input port, which reduces the effective path length through the lossy dielectric substrate.

Table I compares the key parameters of the proposed CPPLWA with those reported for several previously presented CP array antennas. In all cases, the size is provided with respect to the free-space wavelength $\left(\lambda_{0}\right)$ at each corresponding central frequency, and the gain column indicates the minimum gain within the return loss bandwidth. After a quick analysis of Table I, it can be seen that, the impedance bandwidth (RLBW) and maximum gain of the CP-PLWA on PGL are comparable to those of the compared antennas. The axial ratio bandwidth (ARBW) of the proposed antenna is narrower, but its radiation efficiency is higher than in the other CP antennas due to the use of a PGL as a constitutive transmission line.

\section{CONCLUSIONS}

In this letter, a low-loss periodic leaky-wave antenna with circular polarization has been proposed. Measurements of a fabricated prototype have shown good results in the band 38$41 \mathrm{GHz}$, with the typical steering behavior of leaky-wave antennas in both the radiation and AR patterns. The measured radiation efficiency of the antenna is above $90 \%$ due to the use of a PGL as a constitutive transmission line.

The proposed antenna represents a good candidate to be used in radar applications due to its frequency-steering behavior and good AR level. Also, the high gain and efficiency of the CP-PLWA might be used in a $60 \mathrm{GHz}$ version of the antenna to improve the poor communication link budget.

\section{REFERENCES}

[1] J. Volakis, Antenna Engineering Handbook. McGraw Hill, 2009.

[2] D. Navarro Mendez, L. Carrrera Suarez, and M. Escudero, "Circular polarization patch antenna with low axial ratio in a large beamwidth," in 7th European Conference on Antennas and Propagation (EuCAP), April 2013, pp. 3330-3333.

[3] M. Chen and C.-C. Chen, "A compact dual-band GPS antenna design," IEEE Antennas and Wireless Propagation Letters, vol. 12, pp. 245-248, 2013.

[4] K. Chan, A. Tan, and K. Rambabu, "Circularly polarized ultra-wideband radar system for vital signs monitoring," IEEE Transactions on Microwave Theory and Techniques, vol. 61, no. 5, pp. 2069-2075, 2013.

[5] A. Weily and Y. Guo, "Circularly polarized ellipse-loaded circular slot array for millimeter-wave WPAN applications," IEEE Transactions on Antennas and Propagation, vol. 57, no. 10, pp. 2862-2870, Oct 2009.

[6] M. Li and K.-M. Luk, "Low-cost wideband microstrip antenna array for 60-GHz applications," IEEE Transactions on Antennas and Propagation, vol. 62, no. 6, pp. 3012-3018, June 2014.

[7] T. R. Cameron, A. T. Sutinjo, and M. Okoniewski, "A circularly polarized broadside radiating Herringbone array design with the leaky-wave approach," IEEE Antennas and Wireless Propagation Letters, vol. 9, pp. $826-829,2010$

[8] Y. Lang, S.-W. Qu, and J.-X. Chen, "Wideband circularly polarized substrate integrated cavity-backed antenna array," IEEE Antennas and Wireless Propagation Letters, vol. 13, pp. 1513-1516, 2014.

[9] R. Garg, Microstrip antenna design handbook. Artech house, 2001.

[10] M. Ishii, T. Fukusako, and A. Alphones, "Design of leaky wave antenna with composite right-/left-handed transmission line structure for circular polarization radiation," Progress In Electromagnetics Research C, vol. 33, pp. 109-121, 2012.

[11] D. Sanchez-Escuderos, M. Ferrando-Bataller, J. Herranz, and M. Cabedo-Fabres, "Periodic leaky-wave antenna on planar Goubau line at millimeter-wave frequencies," IEEE Antennas and Wireless Propagation Letters, vol. 12, pp. 1006-1009, 2013.

[12] G. Goubau, "Surface waves and their application to transmission lines," Journal of Applied Physics, vol. 21, no. 11, pp. 1119-1128, 1950.

[13] D. Pozar, Microwave and RF design of wireless systems. John Wiley \& Sons, Inc. New York, NY, USA, 2000.

[14] Ansys Corporation, "HFSS," 2014, Suite v15, Pittsburg (PA), USA

[15] A. Garcia-Aguilar, J. Inclan-Alonso, L. Vigil-Herrero, J. FernandezGonzalez, and M. Sierra-Pérez, "Low-profile dual circularly polarized antenna array for satellite communications in the X band," IEEE Transactions on Antennas and Propagation, vol. 60, no. 5, pp. 2276-2284, 2012.

[16] J. E. Hansen, Spherical Near-Field Antenna Measurements. Peter Peregrinus Ltd., London, United Kingdom, 1988. 